\title{
Impact of immunosuppressant therapy on new-onset diabetes in liver transplant recipients
}

\author{
This article was published in the following Dove Press journal: \\ Therapeutics and Clinical Risk Management \\ 18 August 2017 \\ Number of times this article has been viewed
}

\author{
Fu-Chao Liu',2,* \\ Huan-Tang Lin ${ }^{1,2, *}$ \\ Jr-Rung $\operatorname{Lin}^{1-3}$ \\ Huang-Ping $\mathrm{Yu}^{1,2}$ \\ 'Department of Anesthesiology, \\ Chang Gung Memorial Hospital, \\ ${ }^{2}$ College of Medicine, ${ }^{3} \mathrm{Clinical}$ \\ Informatics and Medical Statistics \\ Research Center and Graduate \\ Institute of Clinical Medicine, Chang \\ Gung University, Taoyuan, Taiwan \\ *These authors contributed equally \\ to this work
}

\begin{abstract}
This nationwide, population-based study aimed to clarify the effects of immunosuppressive regimens on new-onset diabetes after liver transplantation (NODALT). The National Health Insurance database of Taiwan was explored for patients who received liver transplantation without pre-transplant diabetes from 1998 to 2012. Information regarding clinical conditions and immunosuppressant utilization among these patients was analyzed statistically. Of the 2,140 patients included in our study, 189 (8.8\%) developed NODALT. The pre-transplant risk factors for NODALT were identified as old age, male sex, hepatitis C, alcoholic hepatitis, and immunosuppressant use of tacrolimus (TAC). All patients used corticosteroids as a baseline immunosuppressant. The immunosuppressant regimen of cyclosporine (CsA)+TAC+mycophenolate mofetil (MMF) contributed most to NODALT (adjusted hazard ratio 7.596) in comparison with the regimens of TAC+MMF and CsA+MMF; this regimen also contributed significantly to higher post-transplant bacteremia, urinary tract infection, pneumonia, renal failure, and mortality rate. In conclusion, our analysis confirmed TAC-based immunosuppression contributes to higher NODALT incidence than CsA-based regimen, and TAC-CsA conversion due to any causes might lead to worse clinical outcomes. Clinicians should make better risk stratifications before prescribing immunosuppressants for liver transplant recipients.

Keywords: new-onset diabetes, liver transplantation, immunosuppressant, population-based study, clinical outcome
\end{abstract}

\section{Introduction}

New-onset diabetes after transplantation (NODAT) is a challenging complication after solid organ transplantation, known to increase risk of infection, cardiovascular events, graft failure, and mortality in organ recipients. ${ }^{1-3}$ The incidence of NODAT has been reported to be $14 \%-44 \%, 31 \%-36 \%, 32 \%-40 \%$, and $28 \%-40 \%$ following liver, kidney, lung, and heart transplantations, respectively, according to American Diabetes Association/World Health Organization (ADA/WHO) criteria. ${ }^{1}$ The incidence of NODAT is higher during the early post-transplant period because of the exposure to high doses of corticosteroids, calcineurin inhibitors (CNIs), and long-term physical inactivity. ${ }^{3,4}$ A meta-analysis including 20 studies concluded that hepatitis $\mathrm{C}$ virus infection, impaired fasting glucose, family history of diabetes, male sex, old age, use of tacrolimus (TAC), and obesity are significant risk factors for NODAT. ${ }^{2}$ The pathogenesis of NODAT is generally explained by CNIs inducing pancreatic beta-cell dysfunction and the gluconeogenesis effect of steroids resulting in insulin resistance. ${ }^{3}$ Therefore, customized selection of immunosuppressant regimen should be emphasized to reduce the incidence of NODAT and thus promote overall survival.

The sophistication of immunosuppression accounts for the remarkable transplantation success in the last three decades, with annual solid organ graft survival
Correspondence: Huang-Ping Yu Department of Anesthesiology, Chang Gung Memorial Hospital, 5 Fu-Shin Street, Kwei-Shan, Taoyuan 333, Taiwan Phone +886 3328 I 200 ext 2324 Fax +886 $3328 \quad 200$ ext 2787 Email yuhp200I@adm.cgmh.org.tw 
rates exceeding $90 \%$ in recent years. ${ }^{5}$ The KDIGO practice guidelines for kidney transplant recipients in 2009 recommended triple immunosuppressant therapy with CNIs (TAC or cyclosporine [CsA]), mycophenolate mofetil (MMF), and optional use of steroids as first-line agents. ${ }^{6}$ CNIs are the cornerstone of maintenance immunosuppressive regimens in solid organ transplantation. CNIs prevent lymphocyte proliferation and interleukin (IL)-2 production by inhibiting signal activation within T-cells, thus effectively preventing acute rejection. ${ }^{7,8}$ The introduction of CNIs (CsA in 1970s, TAC in 1990s) greatly improved the outcomes of organ transplantation. The current most commonly used immunosuppressive regimen in liver transplantation consists of IL-2 antagonist (basiliximab or daclizumab) induction with subsequent maintenance with TAC, MMF, and prednisolone, which has been shown to substantially reduce post-transplant biopsy-proven acute rejection. ${ }^{7}$ However, the use of CNIs is associated with adverse effects including hypertension, hyperlipidemia, glucose intolerance, NODAT, neurotoxicity, nephrotoxicity, and de novo malignancy. ${ }^{7,9} \mathrm{CNI}$-induced nephrotoxicity is recognized as an important cause for post-transplant morbidities and graft failure. Attempts to mitigate the adverse effects of CNIs include delayed introduction of CNI, CNI minimization by substitution with MMF or mammalian target of rapamycin inhibitors (mTORIs), or a CNI-free regimen. ${ }^{10,11}$

Since the mid-1990s, TAC has become the first-line immunosuppressant for liver transplantation. ${ }^{7}$ TAC is $100-$ fold more potent than CsA and is currently prescribed for over $96 \%$ of organ recipients at the time of hospital discharge after transplant surgery. ${ }^{7}$ In 2006, the Cochrane systematic review reported significantly reduced mortality rate, graft failure, acute rejection, and steroid-resistant rejection in TAC-based immunosuppression for liver transplant recipients compared to CsAbased immunosuppression; ${ }^{12}$ however, higher incidence rate of NODAT was also reported in TAC-treated recipients. These clinical studies showed that, compared to CsA, TAC has been associated with better graft survival, less acute rejection, lower nephrotoxicity, easier blood pressure control, and favorable cardiovascular profiles, but higher incidence of NODAT. ${ }^{8}$

The choice of immunosuppressive regimen has significant influence on the development of NODAT and the survival outcome of liver transplant recipients; however, large-scale clinical studies addressing this issue are scarce in the literature. Therefore, we conducted this retrospective, nationwide, population-based cohort study, using the National Health Insurance (NHI) database, to investigate the influence of different immunosuppressive regimens on the incidence of NODAT and outcomes of liver transplantation in Taiwan.

\section{Methods}

\section{Data collection}

This retrospective, population-based cohort study was conducted using Taiwan's NHI research database. De-identified and computerized data were provided by the Bureau of NHI, which organizes claims data for the entire NHI system and established the NHI research database. The NHI database contains basic patient information and medical data, such as international clinical diagnostic (ICD) codes. This study was evaluated and approved by the NHI research committee (NHIRD-103-103) and the institutional review board of the Chang Gung Memorial Hospital. No consent was needed since the data were analyzed anonymously.

\section{Patient and immunosuppressive regimen selection}

Figure 1 presents the flowchart of patient selection and identification process for our study. Liver transplant recipients during the period 1998-2012 were identified from the NHI database using the ICD-9 diagnostic codes V427 (liver transplantation status) and 996.82 (complications of transplanted liver). To exclude recipients who did not undergo liver transplantation in Taiwan, we applied the operation codes of liver transplantation $(505,75020 \mathrm{~A}$, or 75020B). Hence, we identified 2,938 patients who received liver transplantation in Taiwan during the period 1998-2012 in the NHI database. In addition, we also excluded 614 patients with the diagnosis of diabetes mellitus before liver transplantation and another 76 patients with unconfirmed pre-transplant or post-transplant diagnosis of diabetes. We defined NODAT as new-onset diabetes occurring 90 days after liver transplantation; therefore, another 108 patients whose post-transplant follow-up duration was less than 90 days were also excluded. The final cohort consisted of 2,140 liver transplant recipients who received liver transplantation in Taiwan during the period 1998-2012 without pre-transplant diabetes.

The NODAT cases in our study were defined as those with the presence of diagnosis code of diabetes (ICD-9 code 250, A181) more than three times either in inpatient or outpatient department after 90 days of liver transplantation. Demographic data, medical conditions, and mortality rates of recipients with or without NODAT were identified and compared. The following pre-transplant medical conditions were compared among these recipients: hepatitis $\mathrm{B}$, hepatitis $\mathrm{C}$, alcoholic hepatitis, liver cirrhosis, hepatocellular carcinoma, other malignancy, hypertension, coronary heart disease, obesity, coagulopathy, chronic 


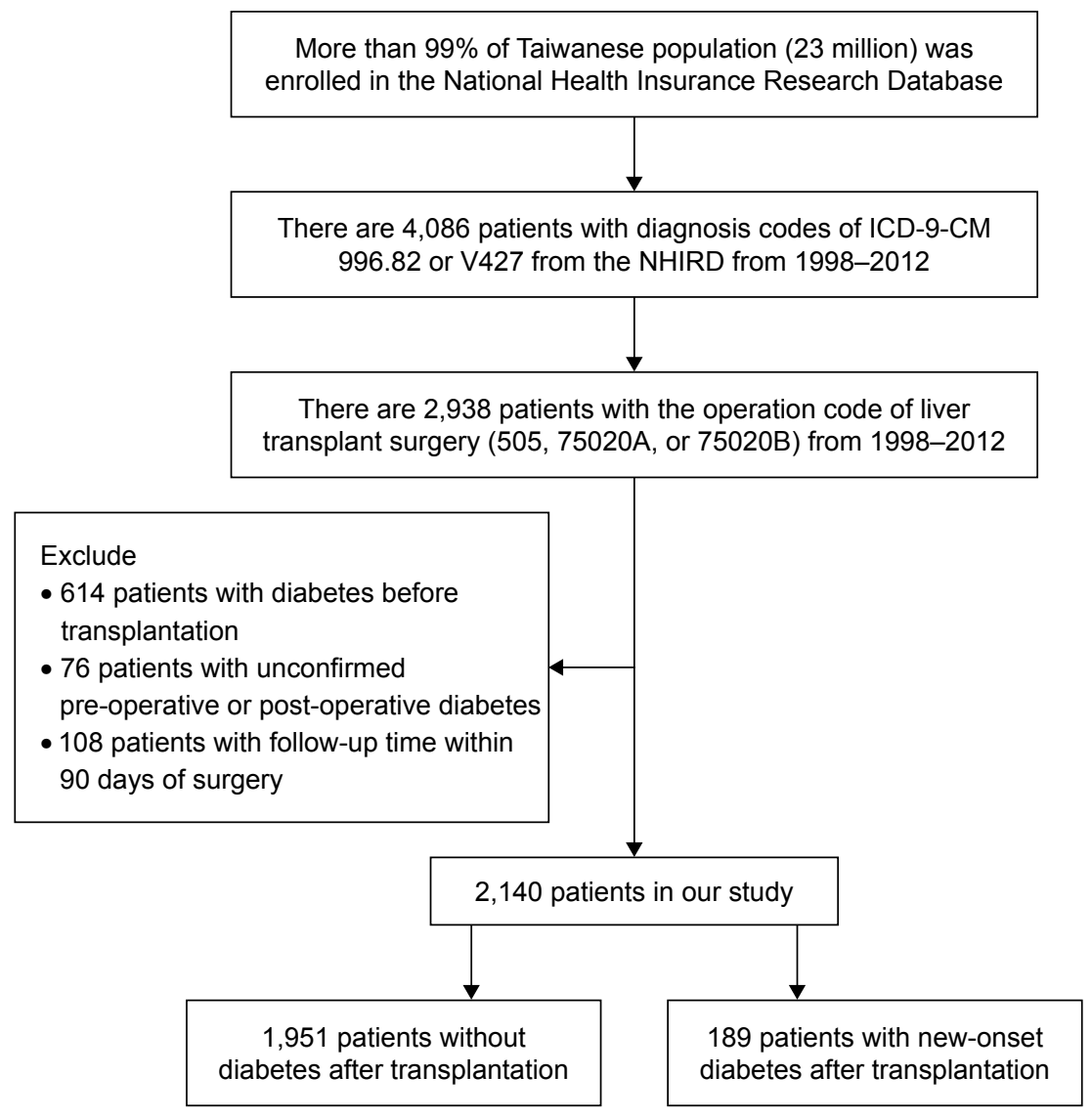

Figure I Study design and flowchart of patient selection.

Abbreviations: ICD, international clinical diagnostic; NHIRD, National Health Insurance Research Database.

kidney disease, and hyperlipidemia. Mortality was identified by the presence of health insurance death codes or termination of NHI.

The utilization of five commonly prescribed immunosuppressants (CsA, TAC, MMF, sirolimus, and everolimus) among liver transplant recipients was investigated and compared. Steroids, as an important component of immunosuppressive therapy, were given for over $99 \%$ of liver transplant recipients in our cohort (prednisolone: 2,935/ $2,938=99.90 \%$; methylprednisolone: $2,924 / 2,938=99.52 \%$ ), and thus were not enlisted for comparison. In our cohort using NHI database, a specific immunosuppressant was defined as part of maintenance regimen if it was identified in more than $10 \%$ of all immunosuppressant prescriptions. As a result, the three most commonly used immunosuppressive regimens were identified as TAC+MMF (64.5\%), CsA+MMF (7.6\%), and CsA+TAC+MMF (3.8\%). Then we evaluated and compared the demographic data, pretransplant medical conditions, incidence of NODAT, and survival outcomes in liver transplant recipients with different immunosuppressant regimens.

\section{Measurements}

The primary outcome in our study was the comparison of pre-transplant medical conditions of liver transplant recipients with or without NODAT. The secondary outcome was the correlation of different immunosuppressive regimens with NODAT and outcomes of liver transplantation. We also compared the incidence of post-transplant infection, cardiovascular events, de novo malignancies, and mortality rates occurring 6 months after liver transplantation for different immunosuppressant regimens. We used Kaplan-Meier survival analysis to clarify the association between different immunosuppressant regimens and their post-transplant survival rate.

\section{Statistical analysis}

Demographic data and medical conditions were analyzed by chi-square or Fisher's exact test for univariate comparisons between categorical variables and Student's $t$-test for continuous variables. Risk factors for NODAT were evaluated using Cox proportional hazard regression model. The hazard ratio of NODAT for each regimen was adjusted by 
significant pre-transplant risk factors of NODAT including age, sex, hepatitis C, and alcoholic hepatitis. Kaplan-Meier method with log-rank test was used to compare overall survival during follow-up period among different regimens. All statistical analysis was performed with SAS software (version 9.3; SAS Institute Inc., Cary, NC, USA), and the tests were two-tailed with $p$-value $<0.05$ considered statistically significant.

\section{Results}

\section{Correlation of comorbidities and immunosuppressant with NODAT}

Our study included 2,140 patients, of whom 189 patients $(8.8 \%)$ developed NODAT. Liver transplant recipients with NODAT were more likely to be older $(p<0.001)$ and male ( $p=0.0373$ ) than recipients without NODAT (Table 1). More patients were found to have pre-transplant hepatitis $\mathrm{C}$ (26.98\% vs $18.81 \%, p=0.0068)$ and alcoholic hepatitis ( $25.60 \%$ vs $16.61 \%, p=0.0023$ ). The analysis of association between five commonly used immunosuppressant prescriptions and NODAT showed that TAC (95.77\% vs $86.67 \%$, $p=0.0003)$ and MMF (82.54\% vs $75.45 \%, p=0.0291)$ had significantly higher correlations with NODAT than the others (Table 2).

Table I Pre-transplant risk factors for NODALT

\begin{tabular}{|c|c|c|c|c|c|}
\hline \multirow{2}{*}{$\begin{array}{l}\text { Risk factors } \\
\\
\text { Age }\end{array}$} & \multicolumn{2}{|c|}{$\begin{array}{l}\text { Recipients } \\
\text { without } \\
\text { NODALT } \\
(n=I, 95 I)\end{array}$} & \multicolumn{2}{|c|}{$\begin{array}{l}\text { Recipients } \\
\text { with } \\
\text { NODALT } \\
(n=189) \\
\end{array}$} & \multirow{2}{*}{$\begin{array}{c}p \text {-value } \\
<0.00 \text { I* }^{*}\end{array}$} \\
\hline & 43.28 & $( \pm 19.77)$ & 49.89 & $( \pm 9.76)$ & \\
\hline Sex & & & & & $0.0373 *$ \\
\hline Female & 618 & (31.68\%) & 46 & $(24.34 \%)$ & \\
\hline Male & 1,333 & $(68.32 \%)$ & 143 & (75.66\%) & \\
\hline \multicolumn{6}{|l|}{ Medical conditions } \\
\hline Hepatitis B & 916 & $(46.95 \%)$ & 91 & $(48.15 \%)$ & 0.7527 \\
\hline Hepa & 367 & (18.8I\%) & 51 & $(26.98 \%)$ & $0.0068^{*}$ \\
\hline Alcoh & 324 & (16.61\%) & 48 & $(25.40 \%)$ & $0.0023^{*}$ \\
\hline Liver cirrhosis & 1,600 & (82.01\%) & 164 & (86.77\%) & 0.1004 \\
\hline Liver cancer & 838 & $(42.95 \%)$ & 89 & (47.09\%) & 0.2730 \\
\hline Non-liver malignancy & 47 & $(2.41 \%)$ & 7 & $(3.70 \%)$ & 0.3245 \\
\hline Hypert & 296 & $(15.17 \%)$ & 19 & $(I 0.05 \%)$ & 0.0579 \\
\hline $\begin{array}{l}\text { Coronary artery } \\
\text { disease }\end{array}$ & 98 & $(5.02 \%)$ & 14 & $(7.41 \%)$ & 0.1599 \\
\hline Obesity & 2 & $(0.10 \%)$ & 0 & $(0)$ & 1.0000 \\
\hline Coagulopathy & 271 & (I3.89\%) & 36 & (I9.05\%) & 0.0535 \\
\hline Chronic renal failure & 54 & $(2.77 \%)$ & 7 & $(3.70 \%)$ & 0.4604 \\
\hline Hyperlipidemia & 138 & (7.07\%) & 16 & $(8.47 \%)$ & 0.4794 \\
\hline
\end{tabular}

Notes: New-onset diabetes after liver transplantation (NODALT) was defined as new-onset diabetes occurring 90 days after liver transplantation. Significant risk factors for NODALT included older age, male sex, hepatitis C, and alcoholic hepatitis. The values given for the risk factor "Age" represent mean age (years) \pm standard deviation; $* p<0.05$.
Table 2 Association of immunosuppressant utilization with NODALT

\begin{tabular}{|c|c|c|c|c|c|}
\hline \multirow{2}{*}{$\begin{array}{l}\text { Immunosuppressant } \\
\text { Cyclosporine (CsA) }\end{array}$} & \multicolumn{2}{|c|}{$\begin{array}{l}\text { Recipients } \\
\text { without } \\
\text { NODALT } \\
(\mathrm{n}=\mathrm{I}, 95 \mathrm{I})\end{array}$} & \multicolumn{2}{|c|}{$\begin{array}{l}\text { Recipients } \\
\text { with } \\
\text { NODALT } \\
(n=189)\end{array}$} & \multirow{2}{*}{$\begin{array}{l}p \text {-value } \\
0.8816\end{array}$} \\
\hline & 363 & (I8.6I\%) & 36 & $(19.05 \%)$ & \\
\hline Tacrolimus (TAC) & 1,691 & (86.67\%) & 181 & (95.77\%) & $0.0003 *$ \\
\hline $\begin{array}{l}\text { Mycophenolate mofetil } \\
\text { (MMF) }\end{array}$ & 1,472 & (75.45\%) & 156 & (82.54\%) & $0.0291 *$ \\
\hline Sirolimus & 376 & $(19.27 \%)$ & 40 & $(21.16 \%)$ & 0.5303 \\
\hline Everolimus & 165 & $(8.46 \%)$ & 13 & $(6.88 \%)$ & 0.4529 \\
\hline
\end{tabular}

Notes: Immunosuppressant utilization was defined as the presence of drug code in more than $10 \%$ of the total immunosuppressant prescriptions. Tacrolimus and mycophenolate mofetil had significant correlations with the incidence of NODALT; $* p<0.05$.

Abbreviation: NODALT, new-onset diabetes after liver transplantation.

\section{Demographic data of patients using different regimens}

Demographic data of patients using different immunosuppressant regimens are listed in Table 3. Patients on regimen TAC+MMF or CsA+TAC+MMF were significantly older and male, and had more pre-transplant diseases, including hepatitis $\mathrm{B}$, hepatitis $\mathrm{C}$, alcoholic hepatitis, liver cancer, hypertension, and coronary artery disease, compared to those on regimen CsA+MMF. Besides, the incidence of NODAT was observed to be the highest in regimen $\mathrm{CsA}+\mathrm{TAC}+\mathrm{MMF}$ (28.4\%) followed by regimen TAC+MMF (9.2\%) and regimen CsA+MMF $(3.7 \%)(p<0.0001)$.

\section{Immunosuppressant regimens and NODAT}

Table 4 shows that these immunosuppressant regimens had different correlations with NODAT. After adjusted by significant pre-transplant medical diseases of NODAT (age, sex, hepatitis $\mathrm{C}$, alcoholic hepatitis), we obtained the adjusted hazard ratios: $\mathrm{CsA}+\mathrm{TAC}+\mathrm{MMF}$ (7.596) $>\mathrm{TAC}+\mathrm{MMF}$ (2.443) $>\mathrm{CsA}+\mathrm{MMF}$ (as standard). Figure 2 shows the incidence rate of NODAT development for different immunosuppressant regimens during the follow-up period after liver transplantation; it reveals that NODAT in most cases developed within 1 year after liver transplantation.

\section{Immunosuppressant regimens and post-transplant outcomes}

Post-transplant outcomes for different regimens were measured as infections (bacteremia, pneumonia, urinary tract infection [UTI]), cardiovascular events (myocardial infarction, cerebral stroke accident), de novo malignancies 
Table 3 Demographic data of recipients using different immunosuppressant regimens

\begin{tabular}{|c|c|c|c|c|}
\hline Demographic data & $\begin{array}{l}\text { TAC+MMF } \\
(\mathbf{N}=1,380)\end{array}$ & $\begin{array}{l}\text { CsA+MMF } \\
(\mathrm{N}=163)\end{array}$ & $\begin{array}{l}\text { CsA+TAC+MMF } \\
(\mathbf{N}=\mathbf{8} \mathrm{I})\end{array}$ & $p$-value \\
\hline Age & $49.83( \pm 12.07)$ & $27.04( \pm 24.01)$ & $42.56( \pm 18.45)$ & $<0.000 I^{*}$ \\
\hline Sex & & & & $<0.000$ I* \\
\hline Female & $375(27.17 \%)$ & $72(44.17 \%)$ & $25(30.86 \%)$ & \\
\hline Male & I,005 (72.83\%) & 91 (55.83\%) & $56(69.14 \%)$ & \\
\hline \multicolumn{5}{|l|}{ Pre-transplant diseases } \\
\hline Alcoholic hepatitis & 280 (20.29\%) & 14 (8.59\%) & II (I3.58\%) & $0.0007 *$ \\
\hline \multirow[t]{2}{*}{ Hepatitis B } & $762(55.22 \%)$ & 42 & $29(35.80 \%)$ & $<0.000$ I* \\
\hline & & $(25.77 \%)$ & & \\
\hline Hepatitis C & $286(20.72 \%)$ & $22(13.5 \%)$ & $23(28.4 \%)$ & $0.0177 *$ \\
\hline Esophageal varices & 735 (53.26\%) & 75 (46.0I\%) & $36(44.44 \%)$ & 0.0793 \\
\hline Ascites & 699 (50.65\%) & $86(52.76 \%)$ & 40 (49.38\%) & 0.8488 \\
\hline Liver cancer & $690(50.00 \%)$ & $30(18.40 \%)$ & 31 (38.27\%) & $<0.000$ I* \\
\hline Non-liver malignancy & 33 (2.39\%) & 5 (3.07\%) & $2(2.47 \%)$ & 0.8705 \\
\hline Hypertension & 237 (17.17\%) & $12(7.36 \%)$ & $12(14.81 \%)$ & $0.0052^{*}$ \\
\hline Coronary artery disease & $88(6.38 \%)$ & $\mathrm{I}(0.61 \%)$ & $3(3.70 \%)$ & $0.0079 *$ \\
\hline Chronic renal failure & 44 (3.19\%) & $4(2.45 \%)$ & I (I.24\%) & 0.5503 \\
\hline \multicolumn{5}{|l|}{ End point } \\
\hline New-onset diabetes & 127 (9.2\%) & $6(3.68 \%)$ & $23(28.4 \%)$ & $<0.000$ I* \\
\hline
\end{tabular}

Notes: Considering pre-transplant comorbidities, liver transplant recipients of regimen TAC+MMF were of significantly older age, male sex, alcoholic hepatitis, hepatitis B, hepatitis $C$, liver cancer, hypertension, and coronary artery disease compared to regimen CsA+MMF. Regarding NODALT incidence, CsA+TAC+MMF regimen had the highest correlation while CsA+MMF regimen had the lowest risk. The values given for "Age" represent mean age (years) \pm standard deviation; * $p<0.05$.

Abbreviations: NODALT, new-onset diabetes after liver transplantation; TAC, tacrolimus; MMF, mycophenolate mofetil; CsA, cyclosporine; DM, diabetes mellitus.

(liver cancer, non-liver malignancy), and death occurring 6 months after liver transplantation. The immunosuppressant regimen CsA+TAC+MMF was associated with the worst outcomes, consisting of higher incidence of bacteremia $(p<0.001)$, pneumonia $(p=0.036)$, UTI $(p=0.007)$, renal failure $(p=0.009)$, and mortality rate $(p=0.012)$. There was no significant difference in post-transplant cardiovascular events or de novo malignancies between these regimens (Table 5). The Kaplan-Meier analysis of post-transplant overall survival for liver transplant recipients with different regimens

Table 4 The Cox proportional hazard model of NODALT between different immunosuppressant regimens

\begin{tabular}{|c|c|c|c|}
\hline $\begin{array}{l}\text { Immunosuppressant } \\
\text { regimen }\end{array}$ & $\begin{array}{l}\text { TAC+MMF } \\
(\mathbf{N}=I, 380)\end{array}$ & $\begin{array}{l}\text { CsA+MMF } \\
(N=163)\end{array}$ & $\begin{array}{l}\text { CsA+TAC+MMF } \\
(\mathbf{N}=\mathbf{8} \mathrm{I})\end{array}$ \\
\hline New-onset diabetes & 127 (9.2\%) & $6(3.7 \%)$ & $23(28.4 \%)$ \\
\hline \multicolumn{4}{|l|}{ Unadjusted } \\
\hline Hazard ratio & 3.843 & I & 10.391 \\
\hline $95 \% \mathrm{Cl}$ & I.680-8.792 & - & $4.226-25.549$ \\
\hline$p$-value ${ }^{a}$ & $0.0014^{*}$ & - & $<0.000 I^{*}$ \\
\hline \multicolumn{4}{|l|}{ Adjusted } \\
\hline Hazard ratio & 2.443 & I & 7.596 \\
\hline $95 \% \mathrm{Cl}$ & I.038-5.748 & - & $3.054-18.898$ \\
\hline$p$-value ${ }^{a}$ & $0.0408^{*}$ & - & $<0.000 I^{*}$ \\
\hline
\end{tabular}

Notes: Hazard ratio was adjusted by age, sex, hepatitis $C$, and alcoholic hepatitis. The adjusted hazard ratios of NODALT for different regimens were as follows: $\mathrm{CsA}+\mathrm{TAC}+\mathrm{MMF}(7.596)>\operatorname{TAC}+\mathrm{MMF}(2.443)>\mathrm{CsA}+\mathrm{MMF}$ (as standard); '-' indicates CsA+MMF were used as standard for $95 \% \mathrm{Cl}$ and $p$-value. ${ }^{a} p$-value was calculated using the Cox proportional hazard model; ${ }^{*} p<0.05$.

Abbreviations: NODALT, new-onset diabetes after liver transplantation; TAC, tacrolimus; MMF, mycophenolate mofetil; CsA, cyclosporine; DM, diabetes mellitus. during the follow-up period is shown in Figure 3. This figure shows that recipients treated with regimen $\mathrm{CsA}+\mathrm{TAC}+\mathrm{MMF}$ were associated with the worst overall survival among all the regimens $(\log -\operatorname{rank} p=0.0427)$.

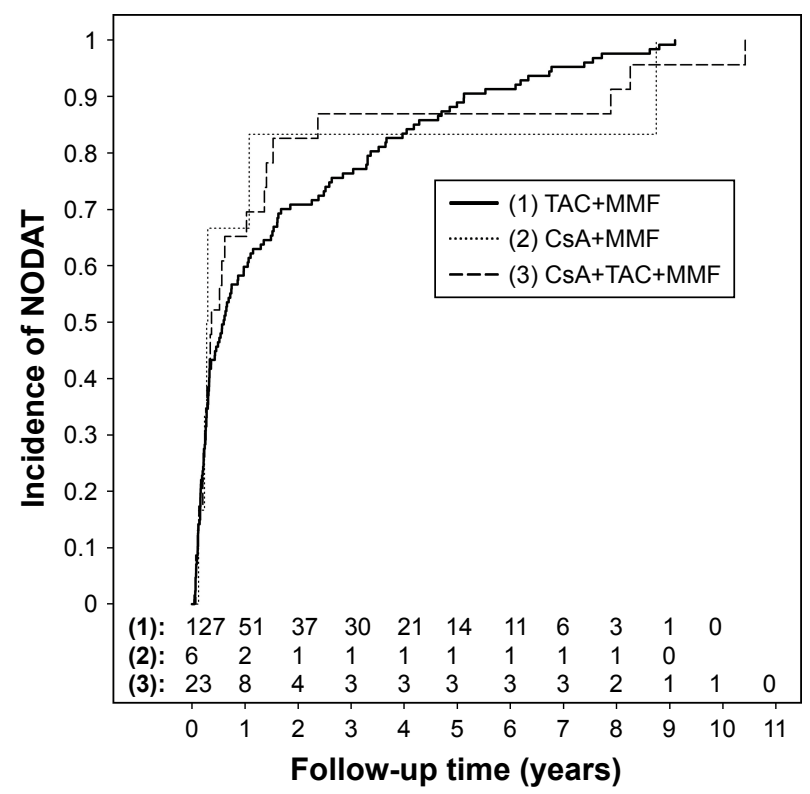

Figure 2 Immunosuppressive regimens and development of NODAT. Notes: NODAT was defined as new-onset diabetes if it occurred 90 days after liver transplantation. The median time of developing NODAT was 0.5996 years with TAC+MMF, 0.2888 years with CsA+MMF, and 0.3696 years with CsA+TAC+MMF regimens.

Abbreviations: NODAT, new-onset diabetes after transplantation; TAC, tacrolimus; MMF, mycophenolate mofetil; CsA, cyclosporine. 
Table 5 The post-transplant outcomes between different immunosuppressant regimens

\begin{tabular}{|c|c|c|c|c|}
\hline $\begin{array}{l}\text { Post-transplant } \\
\text { complications }\end{array}$ & $\begin{array}{l}\text { TAC+MMF } \\
(\mathrm{N}=1,380)\end{array}$ & $\begin{array}{l}\text { CsA+MMF } \\
(\mathrm{N}=163)\end{array}$ & $\begin{array}{l}\text { CsA+TAC+MMF } \\
(\mathrm{N}=8 \mathrm{I})\end{array}$ & p-value \\
\hline Bacteremia & $83(6.01 \%)$ & II (6.75\%) & $16(19.75 \%)$ & $<0.000 I^{*}$ \\
\hline Pneumonia & $13(0.94 \%)$ & $5(3.07 \%)$ & $2(2.47 \%)$ & $0.0362 *$ \\
\hline UTI & 53 (3.84\%) & $8(4.91 \%)$ & $9(11.11 \%)$ & $0.0069 *$ \\
\hline Myocardial infarction & $12(0.87 \%)$ & $0(0 \%)$ & $\mathrm{I}(\mathrm{I} .23 \%)$ & 0.4329 \\
\hline Cerebral stroke accident & $21(1.52 \%)$ & $5(3.07 \%)$ & $2(2.47 \%)$ & 0.2138 \\
\hline Renal failure & 55 (3.99\%) & $8(4.91 \%)$ & $9(11.11 \%)$ & $0.0097^{*}$ \\
\hline Liver cancer & $100(7.25 \%)$ & $4(2.45 \%)$ & $7(8.64 \%)$ & 0.0580 \\
\hline Non-liver malignancy & $53(3.84 \%)$ & $9(5.52 \%)$ & $6(7.41 \%)$ & 0.1989 \\
\hline Death & $158(11.45 \%)$ & $17(10.43 \%)$ & $18(22.22 \%)$ & $0.0120 *$ \\
\hline
\end{tabular}

Notes: Post-transplant clinical outcomes were defined as infection, cardiovascular events, de novo malignancies, and death occurring 6 months after liver transplantation. Liver transplant recipients of regimen CsA+TAC+MMF had the worst clinical outcomes among all the regimens with higher incidence of bacteremia $(p<0.00 \mathrm{I})$, pneumonia $(p=0.036)$, UTI $(p=0.007)$, renal failure $(p=0.009)$, and mortality rate $(p=0.012) ;{ }^{*} p<0.05$.

Abbreviations: TAC, tacrolimus; MMF, mycophenolate mofetil; CsA, cyclosporine; UTI, urinary tract infection.

\section{Discussion}

\section{Interpretation of current results and comparison with previous studies}

In this cohort study, $8.8 \%$ of liver transplant recipients developed NODAT, with the major risk factors being older age, male sex, hepatitis $\mathrm{C}$, alcoholic hepatitis, and the utilization of TAC. Previous studies have showed that NODAT is associated with old age, male sex, high body mass index, viral infection, and impaired fasting glucose; ${ }^{2}$ these results were consistent with our findings, except for obesity. The possible explanation for that insignificant association with obesity might be explained by the relatively low incidence of obesity (only 2 cases in 2,140 recipients) in our cohort, which might be the consequence of undercoding in the NHI coding system of Taiwan.

Regarding the association of immunosuppressants and NODAT, our results showed that the utilization of

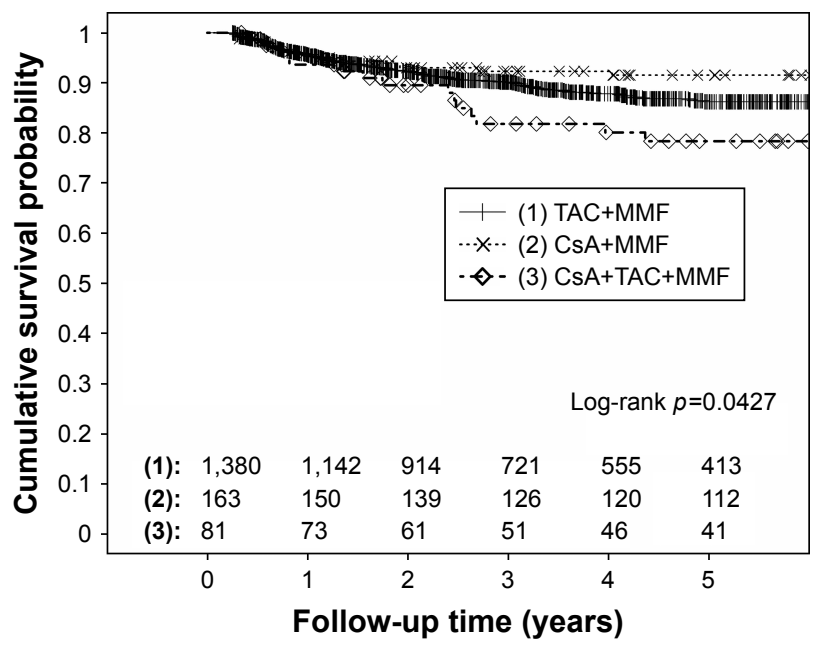

Figure 3 Immunosuppressive regimens and the post-transplant survival curve. Notes: Death was defined as that occurring 6 months after liver transplantation. Liver transplant recipients of CsA+TAC+MMF regimen had the worst survival probability among all the regimens.

Abbreviations: TAC, tacrolimus; MMF, mycophenolate mofetil; CsA, cyclosporine. immunosuppressants TAC and MMF has significant correlation with NODAT. It is well recognized that TAC contributes to higher incidence of NODAT than CsA. While previous studies have reported that MMF has no direct metabolic consequence in organ recipients, its introduction may reduce the total required dosage of steroids and CNIs for immunosuppression. ${ }^{5}$ Therefore, MMF might literally play a protective role against NODAT development in case of immunosuppression with steroids and CNIs. The other immunosuppressants listed in Table 2, sirolimus and everolimus (members of mTORIs), work synergistically with TAC and are generally used in the CNI-minimization regimen to reduce CNI nephrotoxicity due to their non-nephrotoxic properties. In addition, mTOR signaling plays a role in tumor angiogenesis and proliferation, ${ }^{10}$ thus mTORIs have been reported to improve recurrence-free and overall survival in patients undergoing liver transplantation for hepatic cellular carcinoma (HCC) compared with CNIs. ${ }^{13}$ Considering the role of sirolimus in NODAT, clinical results were paradoxical with biphasic effect of enhancing insulin sensitivity in acute treatment but causing insulin resistance after chronic dosing. ${ }^{5}$

The immunosuppressive regimens listed in our study, namely CNIs/MMF plus baseline steroids, were the most used regimens for liver transplant recipients in Taiwan during the 14-year study period. The most commonly used regimen, based on our results, was TAC/MMF plus baseline steroids, which had a modest correlation with NODAT and relatively good clinical outcomes, although its users were of older age and had more pre-transplant diseases. The least used regimen was $\mathrm{CsA}+\mathrm{TAC}+\mathrm{MMF}$, which might be the result of conversion from TAC to CsA due to NODAT or transition from CsA to TAC due to serious rejection. Although the exact clinical scenarios could not be identified based on the NHI database, we found their significant association with higher NODAT 
incidence, more infection events, and higher mortality. The last regimen, CsA+MMF, was used by relatively younger recipients with fewer pre-transplant diseases and was associated with the lowest NODAT incidence, slightly higher renal impairment, and comparable post-transplant overall survival when compared to TAC+MMF regimen.

Previous clinical studies showed higher association of NODAT with regimen containing TAC than CsA. In the Efficacy Limiting Toxicity Elimination (ELITE)-Symphony study of kidney-transplant recipients, ${ }^{14}$ a 12 -month regimen of daclizumab, MMF, and corticosteroids with low-dose TAC provided adequate immunosuppression with better renal function, allograft survival, and less acute rejection, but higher NODAT incidence, as compared with regimens containing either CsA or sirolimus. Another Phase III clinical trial compared the clinical outcomes of TAC vs CsA in kidney transplant recipients showed that regimens TAC/MMF and CsA/MMF were associated with comparable patient and graft survival, but inferior renal function in $\mathrm{CsA} /$ MMF and higher NODAT incidence in TAC/MMF. ${ }^{15}$ These results were compatible with our findings. The highest association of NODAT incidence with regimen $\mathrm{CsA}+\mathrm{TAC}+\mathrm{MMF}$ in our results might be explained by higher percentage of TAC-related NODAT forcing conversion from TAC to CsA. If NODAT developed after standard dosage of TACbased immunosuppression, clinicians might consider TAC minimization or transition to $\mathrm{CsA} .{ }^{16}$ Previous clinical studies showed regression of NODAT incidence in liver transplant recipients after substituting TAC with CsA. ${ }^{17-19}$ However, the possible regression of NODAT in regimen $\mathrm{CsA}+\mathrm{TAC}+\mathrm{MMF}$ was statistically undetectable in our study, since we defined NODAT with the presence of ICD-9 codes.

In view of post-transplant survival outcomes, our retrospective analysis revealed that liver transplant recipients treated with regimen $\mathrm{Cs}+\mathrm{MMF}$ and $\mathrm{TAC}+\mathrm{MMF}$ had comparable survival probability, while those treated with regimen $\mathrm{Cs} A+\mathrm{TAC}+\mathrm{MMF}$ had worse results. Recipients with regimen $\mathrm{Cs}+\mathrm{TAC}+\mathrm{MMF}$ might have undergone conversion from CsA to TAC due to acute rejection episode, which would theoretically translate into more infection events, renal impairment, predisposition to chronic rejection, and consequently higher mortality rates.

The reported measures to reduce incidence of NODAT include regular blood glucose monitoring, rapid steroid reduction, conversion to CsA, TAC-minimization, lifestyle modifications, or CNI-free regimen with belatacept. ${ }^{20}$ Although CNIs remain the cornerstone of immunosuppressive therapy in solid organ transplantation, their dosage should be individualized based on the following guidelines: 1) older recipients receiving CNI reduction and early steroid withdrawal, ${ }^{21}$ 2) recipients with renal insufficiency and NODAT with the preference of minimizing CNI with MMF or everolimus, 3 ) recipients with hepatitis $C$ infection requiring early steroid withdrawal and possible everolimus, and 4) recipients with concomitant HCC with possible benefit from mTORIs. ${ }^{7,10}$

\section{Limitations of this study}

This large, retrospective, nationwide cohort study using the NHI database has some inherent limitations. First, the NHI database contained little information regarding actual clinical situations such as level of glycohemoglobin, fasting glucose, family history, prescription sequence, and drug dosage. Although steroids and CNIs play key roles in the development of NODAT, which might be managed with early steroid withdrawal or CNI minimization nowadays, ${ }^{5}$ our NHI database could only distinguish the existence of prescription codes instead of timely dosage adjustments. Therefore, recipients with regimen $\mathrm{CsA}+\mathrm{TAC}+\mathrm{MMF}$ might have undergone conversion from $\mathrm{CsA}$ to TAC or TAC to CsA, but we could not ascertain the actual situations in the NHI database. Second, we defined NODAT based on the presence of ICD-9 code of diabetes more than three times either in inpatient or outpatient department after 90 days of liver transplantation, while most previous studies defined NODAT with the ADA/WHO criteria. In our NHI system, medical professionals must provide sufficient medical reports to expert panels before approval of waivers. The validity, representativeness, and clinical consistency of this NHI database have been reported in the literature. Therefore, the presented diagnosis of diabetes by the clinicians must be precise (based on ADA/WHO criteria) and consistent, and patients with hyperglycemia or transient surgical diabetes were excluded. Besides, even though our study might underestimate the true incidence of NODAT, the comparison of NODAT incidence between different immunosuppressant regimens yielded meaningful results. Lastly, we defined immunosuppressant utilization by the presence of drug codes in more than $10 \%$ of all immunosuppressant prescriptions to simplify our analysis process. However, an individualized immunosuppressant regimen or regimen conversion might require fewer than $10 \%$ of prescriptions, thus leading to potential statistical bias in our study.

\section{Conclusion}

In this retrospective, nationwide cohort study using NHI database, the pre-transplant risk factors associated with NODALT were identified as old age, male sex, hepatitis $\mathrm{C}$, alcoholic hepatitis, and immunosuppressant use of TAC. 
The most used immunosuppressant regimen for liver transplant recipients during the period 1998-2012 in Taiwan was TAC+MMF plus baseline steroids. Recipients with regimen CsA+TAC+MMF had the highest association with NODAT and worse post-transplant outcomes, including higher incidence of bacteremia, pneumonia, UTI, renal failure, and mortality rate, in comparison with regimen $\mathrm{TAC}+\mathrm{MMF}$ or CsA+MMF. This observation might result from the process of regimen conversion from CsA to TAC due to acute rejection, or conversion from TAC to CsA due to NODAT. Our analysis confirmed TAC-based immunosuppression contributes to higher incidence of NODAT than CsA-based regimen, and TAC-CsA conversion might translate into higher incidence of NODAT and worse clinical outcomes. Therefore, we recommend that clinicians should make better risk stratification before prescribing immunosuppressant regimen for liver transplant recipients.

\section{Acknowledgments}

This study was based on data from the NHI research database, provided by the Bureau of NHI, Department of Health, and managed by the National Health Research Institutes. The interpretation and conclusions contained herein do not represent the views of the Bureau of NHI, Department of Health, or National Health Research Institutes. This work was partially supported by grants from Chang Gung Medical Foundation (project CORPG3E0131).

\section{Author contributions}

F-CL and H-TL contributed toward conceptualization, formal analysis, investigation, and project administration. F-CL was also involved in resource acquisition. H-PY contributed to conceptualization, funding acquisition, investigation, project administration, resource acquisition, and supervision of the project. J-RL was involved in data acquisition, formal analysis, and designing of the methodology. All authors contributed toward data analysis, drafting and revising the paper and agree to be accountable for all aspects of the work.

\section{Disclosure}

The authors report no conflicts of interest in this work.

\section{References}

1. Ling Q, Xu X, Wang B, Li L, Zheng S. The origin of new-onset diabetes after liver transplantation: liver, islets, or gut? Transplantation. 2016;100(4):808-813.
2. Li DW, Lu TF, Hua XW, et al. Risk factors for new onset diabetes mellitus after liver transplantation: a meta-analysis. World J Gastroenterol. 2015;21(20):6329-6340.

3. Kesiraju S, Paritala P, Rao Ch UM, Sahariah S. New onset of diabetes after transplantation - an overview of epidemiology, mechanism of development and diagnosis. Transpl Immunol. 2014;30(1):52-58.

4. Liu FC, Lin JR, Chen HP, Tsai YF, Yu HP. Prevalence, predictive factors, and survival outcome of new-onset diabetes after liver transplantation: a population-based cohort study. Medicine. 2016;95(25):e3829.

5. Bamgbola O. Metabolic consequences of modern immunosuppressive agents in solid organ transplantation. Ther Adv Endocrinol Metab. 2016;7(3):110-127.

6. Kidney Disease: Improving Global Outcomes Transplant Work Group. KDIGO clinical practice guideline for the care of kidney transplant recipients. Am J Transplant. 2009;9(Suppl 3):S1-S155.

7. de Mare-Bredemeijer EL, Metselaar HJ. Optimization of the use of calcineurin inhibitors in liver transplantation. Best Pract Res Clin Gastroenterol. 2012;26(1):85-95.

8. Malvezzi P, Rostaing L. The safety of calcineurin inhibitors for kidney-transplant patients. Expert Opin Drug Saf. 2015;14(10): 1531-1546.

9. Webber AB, Vincenti F. An update on calcineurin inhibitor-free regimens: the need persists, but the landscape has changed. Transplantation. 2016;100(4):836-843.

10. Moini M, Schilsky ML, Tichy EM. Review on immunosuppression in liver transplantation. World J Hepatol. 2015;7(10):1355-1368.

11. Neuberger J. An update on liver transplantation: a critical review. J Autoimmun. 2016;66:51-59.

12. Haddad EM, McAlister VC, Renouf E, Malthaner R, Kjaer MS, Gluud LL. Cyclosporin versus tacrolimus for liver transplanted patients. T Cochrane Database Syst Rev. 2006;(4):CD005161.

13. Geissler EK, Schnitzbauer AA, Zulke C, et al. Sirolimus use in liver transplant recipients with hepatocellular carcinoma: a randomized, multicenter, open-label phase 3 trial. Transplantation. 2016;100(1): $116-125$.

14. Ekberg H, Tedesco-Silva H, Demirbas A, et al. Reduced exposure to calcineurin inhibitors in renal transplantation. N Engl J Med. 2007; 357(25):2562-2575.

15. Silva HT Jr, Yang HC, Meier-Kriesche HU, et al. Long-term follow-up of a phase III clinical trial comparing tacrolimus extendedrelease/MMF, tacrolimus/MMF, and cyclosporine/MMF in de novo kidney transplant recipients. Transplantation. 2014;97(6):636-641.

16. Song JL, Gao W, Zhong Y, et al. Minimizing tacrolimus decreases the risk of new-onset diabetes mellitus after liver transplantation. World $J$ Gastroenterol. 2016;22(6):2133-2141.

17. Lorho R, Hardwigsen J, Dumortier J, et al. Regression of new-onset diabetes mellitus after conversion from tacrolimus to cyclosporine in liver transplant patients: results of a pilot study. Clin Res Hepatol Gastroenterol. 2011;35(6-7):482-488.

18. Dumortier J, Bernard S, Bouffard Y, Boillot O. Conversion from tacrolimus to cyclosporine in liver transplanted patients with diabetes mellitus. Liver Transpl. 2006;12(4):659-664.

19. Ramos-Cebrian M, Torregrosa JV, Gutierrez-Dalmau A, Oppenheimer F, Campistol JM. Conversion from tacrolimus to cyclosporine could improve control of posttransplant diabetes mellitus after renal transplantation. Transplant Proc. 2007;39(7):2251-2253.

20. Palepu S, Prasad GV. New-onset diabetes mellitus after kidney transplantation: current status and future directions. World J Diabetes. 2015;6(3):445-455.

21. Krenzien F, ElKhal A, Quante M, et al. A rationale for age-adapted immunosuppression in organ transplantation. Transplantation. 2015; 99(11):2258-2268. 
Therapeutics and Clinical Risk Management

Dovepress

\section{Publish your work in this journal}

Therapeutics and Clinical Risk Management is an international, peerreviewed journal of clinical therapeutics and risk management, focusing on concise rapid reporting of clinical studies in all therapeutic areas, outcomes, safety, and programs for the effective, safe, and sustained use of medicines. This journal is indexed on PubMed Central, CAS,

EMBase, Scopus and the Elsevier Bibliographic databases. The manuscript management system is completely online and includes a very quick and fair peer-review system, which is all easy to use. Visit http://www.dovepress.com/testimonials.php to read real quotes from published authors.

Submit your manuscript here: http://www.dovepress.com/therapeutics-and-clinical-risk-management-journal 